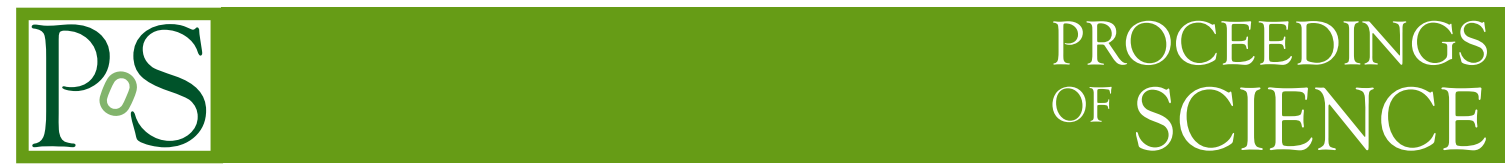

\title{
Recent result of nucleon time-like form factors at BESIII
}

\section{Destefanis ${ }^{a, *}$ for the BESIII Collaboration}

${ }^{a}$ Università degli Studi di Torino and INFN, Via Giuria 1, Torino, Italy

E-mail: marco.destefanis@to.infn.it

The BESIII experiment, hosted at the IHEP of Beijing, has collected the world largest data sample in the charmonium energy region. One of the most important physics goals of BESIII is the investigation of the QCD prediction. QCD can be accessed in a unique way by means of hadron spectroscopy. Charmonium decays provide an excellent scenario for studying nucleons and their properties. Of outmost importance is a deep understanding of the structure and the internal dynamics of nucleons, which can be accessed by means of the Form Factors (FFs). The most recent results for proton and neutron FFs from BESIII will be discussed.

\footnotetext{
*** The European Physical Society Conference on High Energy Physics (EPS-HEP2021), *** *** 26-30 July $2021 * * *$

*** Online conference, jointly organized by Universität Hamburg and the research center DESY ***
}

${ }^{*}$ Speaker 


\section{Introduction}

The complete understanding of strong interactions requires a precise investigation of the fundamental building blocks of atomic matters, the nucleons. Electromagnetic probes can improve our knowledge on internal structure and dynamics of nucleons, taking advantage from the electric and magnetic form factors (FFs), $G_{E}\left(q^{2}\right)$ and $G_{M}\left(q^{2}\right)$, the simplest nucleon structure observables. Elastic scattering experiments (Fig.1a) allow to investigate the space-like (SL) kinematic region $\left(q^{2}<0\right)$, known at few percent precision. Annihilation processes (Fig. 1b) grant access to the timelike (TL) kinematic region $\left(q^{2}>0\right)$, where in most cases only the effective form factor $\left(G_{e f f}\right)$ or the $G_{E} / G_{M}$ ratio were extracted with a precision of the $10 \%$ level. Dispersion relations allow to relate SL and TL FFs [2]. Nucleon FFs in the TL region can be associated with the time evolution of the charge and magnetic distributions inside the nucleon [3].

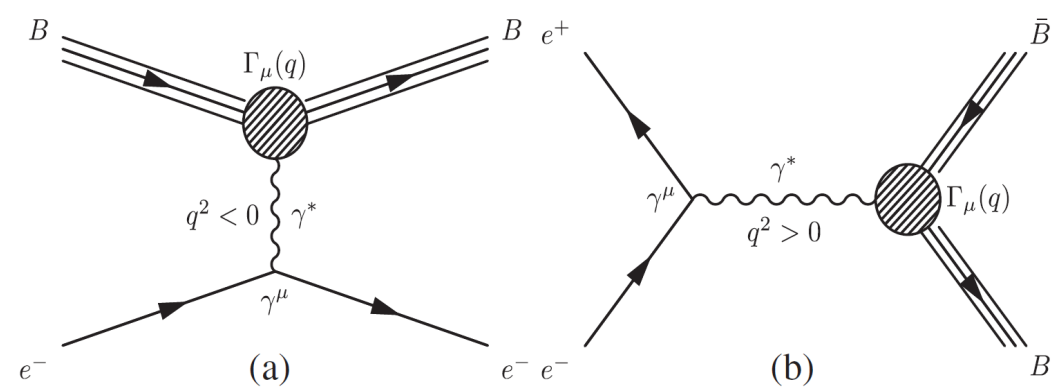

Figure 1: Lowest order Feynman diagrams for elastic electron-baryon scattering (a) and for annihilation process (b), where B indicates a baryon [1].

For annihilation processes, under the assumption of one virtual photon exchange, in the $e^{+} e^{-}$ center of mass frame the differential cross section can be written as:

$$
\frac{d \sigma_{B}}{d \Omega}=\frac{\alpha^{2} \beta^{2} C}{4 q^{2}}\left[\left(1+\cos ^{2} \vartheta\right)\left|G_{M}\right|^{2}+\frac{1}{\tau} \sin ^{2} \vartheta\left|G_{E}\right|^{2}\right],
$$

where $q^{2}$ is the transfered center of mass energy, $\alpha$ is the fine strucure constant, $\beta=\sqrt{1 /(1-\tau)}$, with $\tau=q^{2} / 4 M^{2}$ and $M$ is the baryon mass, $C=\frac{y}{1-e^{-y}}$ is the Coulomb factor, with $y=\alpha \pi / \beta$, and $\vartheta$ is the polar angle of the baryon in the $e^{+} e^{-}$rest frame. Moreover, electromagnetic FFs can be measured in a wider energy region when exploiting the initial state radiation (ISR) technique, in which an initial state particle emits a photon before the annihiliation, thus reducing the center of mass energy available. This process allows the investigation of the FFs from threshold up to the center of mass collider energy.

By integrating over the angular distribution, it is possible to obtain the Born cross section as:

$$
\sigma_{\text {Born }}=\frac{2 \pi \alpha^{2} \beta C}{3 q^{2} \tau}\left(2 \tau\left|G_{M}\right|^{2}+\left|G_{E}\right|^{2}\right) .
$$

The effective form factor $\left(G_{e f f}\right)$ is defined as a linear combination of $G_{E}$ and $G_{M}$ FFs, and it is proportional to the square root of the nucleon pair production cross section:

$$
\left|G_{e f f}\right|=\sqrt{\frac{2 \tau\left|G_{M}\right|^{2}+\left|G_{E}\right|^{2}}{2 \tau+1}}
$$




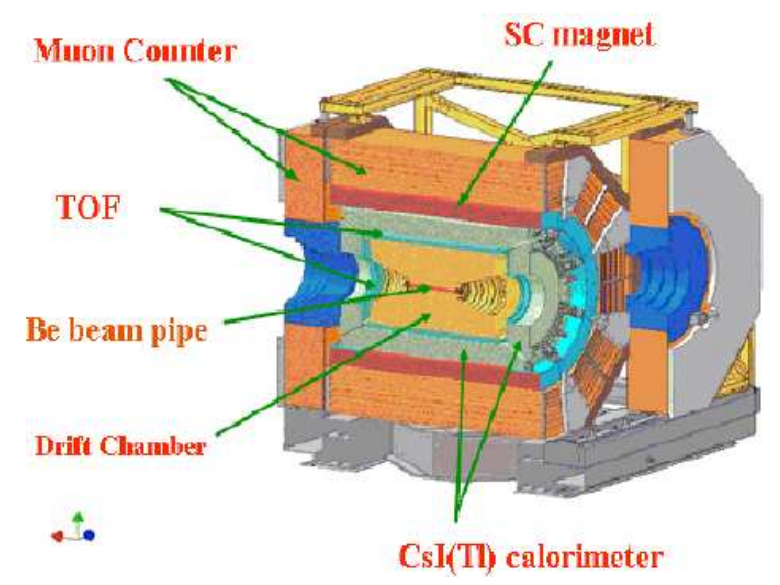

Figure 2: $3 D$ view of the BESIII spectrometer.

The excellent performances of the BEijing Spectrometer III (BESIII) [4], hosted on the BEPCII $\mathrm{e}^{+} \mathrm{e}^{-}$collider at the Institute of High Energy Physics (IHEP) in Beijing, offers the appropriate scenario for FFs measurements. The BEPCII (Beijing Electron-Positron Collider II) operates with beam currents up to $0.93 \mathrm{~A}$, providing an instantaneous luminosity up to $10^{33} \mathrm{~cm}^{-2} \mathrm{~s}^{-1}$. The beam energy (up to $2.473 \mathrm{GeV}$ ) can be tuned according to the required center of mass energy, for example to produce different charmonium resonances. The BESIII spectrometer (Fig.2) is characterized by a shell like structure, which provides a wide geometrical acceptance, of about $93 \%$ of $4 \pi$. At increasing distances from the interaction point, it hosts, inside a $1 \mathrm{~T}$ solenoidal magnetic field, a 43-layer small-celled, helium-based main drift chamber (MDC), a time-of-flight system (TOF) for particle identification, and an electromagnetic calorimeter (EMC) composed of $6240 \mathrm{CsI}$ (Tl) crystals arranged in a cylindrical shape (barrel) plus two end-caps. The magnet iron yoke is segmented to host a muon chamber system (MUC) composed of resistive plate chambers (for a total of 1000 $\mathrm{m}^{2}$ ) arranged in 9 layers in the barrel and 8 layers in the end-caps. The excellent quality of the experimental data collected grant precision studies as well as new physics searches.

\section{Proton Form Factors}

Proton electromagnetic FFs in the time-like region were investigated by BESIII in four separate analyses. Two of them used the scan data, while the others exploited the ISR method both tagging and untagging the ISR photon. The first analysis of an energy scan, aimed to measure the total cross section for the reaction $e^{+} e^{-} \rightarrow p \bar{p}$, was performed using the $157 \mathrm{pb}^{-1}$ data collected at 12 different energy values ranging from 2.2324 to $3.6710 \mathrm{GeV}$ [5]. A second direct scan analysis was performed taking advantage of a $688.5 \mathrm{pb}^{-1}$ more recent high luminosity data set registered at 22 energy values between 2.00 and $3.08 \mathrm{GeV}$ [1]. The ISR analyses, both tagged [6] and untagged [7], are based on the $7.5 \mathrm{fb}^{-1}$ collected at center of mass energies between 3.773 and $4.600 \mathrm{GeV}$. Due to the acceptance region, the two analyses addressed two different energy regions depending on the photon emission polar angle: $\sqrt{s}=2.000-3.800 \mathrm{GeV} / \mathrm{c}$ at small polar angle (SA-ISR), and $\sqrt{s}=1.876-3.000 \mathrm{GeV} / \mathrm{c}$ at large polar angle (LA-ISR). 

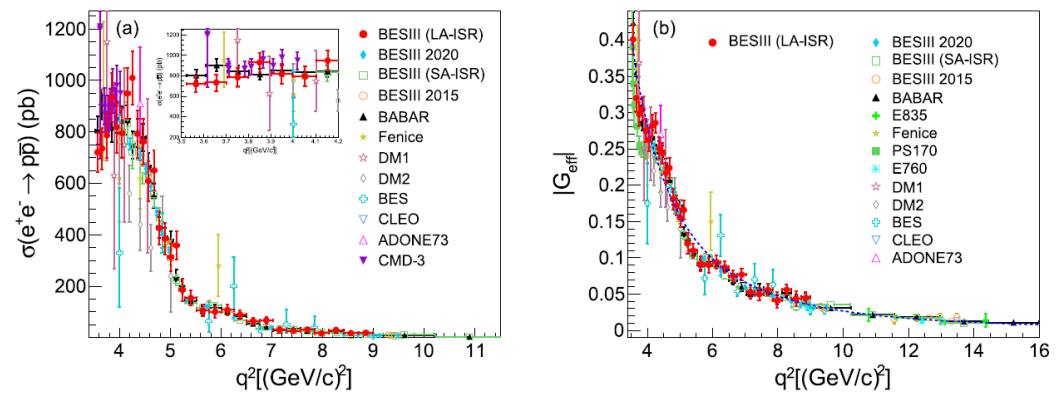

Figure 3: Cross section for the process $e^{+} e^{-} \rightarrow p \bar{p}$ (left) and effective FF of the proton (right) compared with the results from the previous experiments [6].

Cross sections and $G_{\text {eff }}$ results (Fig. 3) show a quite good agreement with most of the data available from the litterature from DM1, DM2, FENICE, BES, PS170, E760, E835, ADONE73, CLEO, and CMD-3 experiments [6]. The relative precision in the determination of the cross section ranges from 3.0 to $12.0 \%$, while for the effective FF it varies between 1.7 and $11.8 \%$.

Figure 4a show the first measurement of $\left|G_{E}\right|$ in the time-like region. Close to the thereshold region, a significant discrepancy between the BaBar and PS170 results on the $\left|G_{E}\right| /\left|G_{M}\right|$ ratio has been observed. The precision of the BESIII measurements, shown in Fig. $4 \mathrm{~b}$ allow to shed light on this puzzle, supporting the BaBar results.

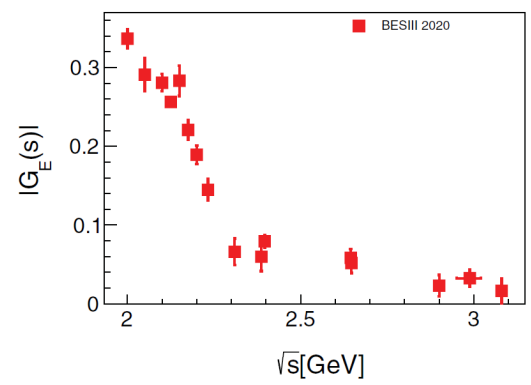

(a)

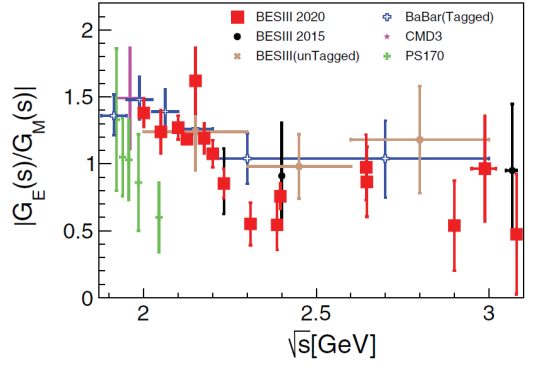

(b)

Figure 4: Electric form factor $\left(\left|G_{E}\right|\right)$ of the proton (a) and $\left|G_{E}\right| /\left|G_{M}\right|$ ratio (b) [1].

\section{Neutron Form Factors}

The measurement of the neutron FFs in $e^{+} e^{-} \rightarrow n \bar{n}$ processes is quite complicated due to difficulties in the detection of the final state particles. For this reason, the observables related to the neutron structure in such process were measured only by the FENICE, DM2, and SND experiments [8]. The high integrated luminosity data samples $\left(647.9 \mathrm{pb}^{-1}\right)$, collected by the BESIII experiment at 18 different center of mass energy values ranging from 2.0 to $3.08 \mathrm{GeV}$, offer a unique opportunity for a precise measurement of the neutron structure [9]. In order to improve the efficiency of $n \bar{n}$ reconstruction, the process $e^{+} e^{-} \rightarrow n \bar{n}$ is investigated by means of three statistically independent signal selections, exploiting a combination of EMC and TOF information. 

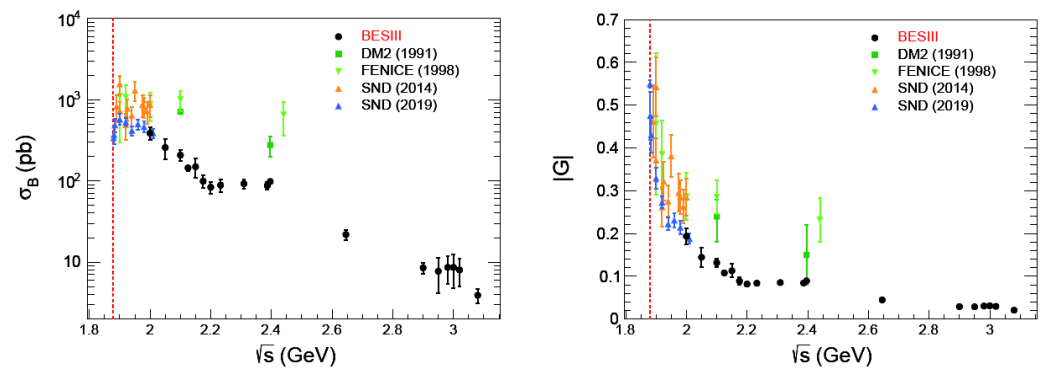

Figure 5: Cross section for the process $e^{+} e^{-} \rightarrow n \bar{n}$ (left) and effective FF of the neutron (right) compared with the results from the previous experiments [9].

In order to take into account the challenging simulation of hadronic interaction with the detector material, the efficiency is determined with data-driven methods. Cross section (left) and effective FF (right) results are shown in Fig. 5, respectively. The statistical precision of the neutron FF ranges between $4.1 \%$ and $43 \%$, improving by a factor 60 with respect the previous measurements from FENICE and DM2 experiments. As it can be observed, at $\sqrt{s}=2.0 \mathrm{GeV}$ our measurements are consistent with the SND data, while at $\sqrt{s}=2.4 \mathrm{GeV}$ there is a $2 \sigma$ difference with the FENICE results.

\section{Oscillation Structure}

An interesting oscillating behavior of the proton $\left|G_{e f f}\right|$ data was pointed out by a recent review of BaBar data [10] after substracting the dipole-like behavior. The small oscillations could be due to re-scattering interference effects in the final state [11] or to the presence of resonant structures [12]. This observation can be reproduced by the discussed analyses for both proton and neutron $\left|G_{e f f}\right|$. A fit with a dipole-like function is performed on the $\left|G_{e f f}\right|$ data. The reduced form factor $G_{o s c}$, after the subtraction of the dipole-like background, can be parametrized as:

$$
G_{o s c}=A \cdot(-B \cdot p) \cdot \cos (C \cdot p+D),
$$

where $p$ is the relative momentum of the nucleon pair, $A$ the normalization, $B$ the inverse oscillation damping, $C$ the momentum frequency, and $D$ the phase. The results of the fit to BESIII proton data are shown in Fig. 6a. With a common momentum frequency $C$, we performed a simultaneous fit to the neutron data from BESIII [9] and proton data from BaBar [10], observing an almost orthogonal periodic behavior for $|G|$ of the neutron, when compared to the one of the proton, as shown in Fig. $6 b$.

\section{Conclusions}

The BESIII experiment provides an excellent scenario for the investigation of the nucleon structure in the time-like region, taking advantage of the high luminosity data samples collected. The cross section and effective form factor measurement precision is improved with respect to the previous experiments. For the proton, $\left|G_{E}\right|$ and $\left|G_{M}\right|$ have been measured for the first time in this 


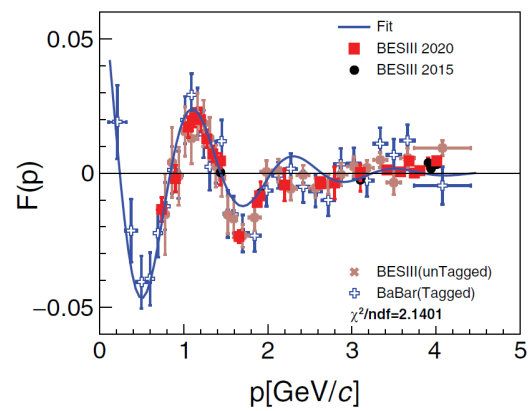

(a)

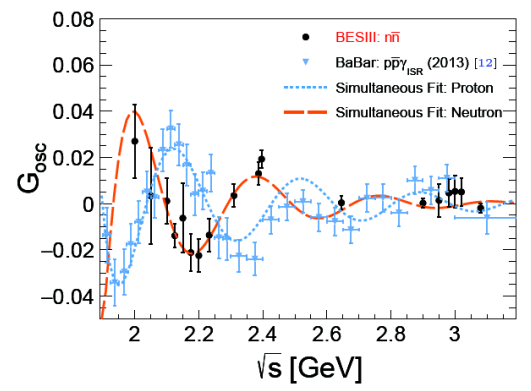

(b)

Figure 6: Effective form factor after the substraction of the dipole-like function for the proton (a) as function of the relative momentum [1] and for the neutron (b) as function of $\sqrt{s}$ [9] compared with BaBar data [10].

region. Moreover, the deviation from the dipole law of the electromagnetic structure observables of the nucleon, observed by BaBar, is confirmed in both proton and neutron data. More experimental data as well as theoretical investigation could shed light on the origin of the oscillation of $\left|G_{e f f}\right|$.

\section{References}

[1] M. Ablikim et al., Phys. Rev. Lett. 124, 042001 (2020), and references therein.

[2] M. Belushkin et al., Phys. Rev. C 75, 035202 (2007).

[3] E.A. Kuraev et al., Phys. Lett. B 712, 240-244 (2012).

[4] D.M. Asner et al., Physics at BES-III, arXiv:0809.1869v1 [hep-ex] (2008).

[5] M. Ablikim et al., Phys. Rev. D 91, 112004 (2015).

[6] M. Ablikim et al., Phys. Lett. B 817, 136328 (2021), and references therein.

[7] M. Ablikim et al., Phys. Rev. D 99, 092002 (2019).

[8] A. Antonelli et al., Nucl. Phys. B 517, 3 (1998).

D. Bisello et al., Z. Phys. C 48, 23 (1990).

M. N. Achasov et al., Phys. Rev. D 90, 112007 (2014).

V. P. Druzhinin, and S.I. Serednyakov, EPJ Web Conf. 212, 07007 (2019).

[9] M. Ablikim et al., arXiv:2103.12486, accepted for publication.

[10] J. P. Lees et al., Phys. Rev. D 88, 032011 (2013).

[11] A. Bianconi, and E. Tomasi-Gustafsson, Phys. Rev. Lett. 114, 232301 (2015).

[12] I.T. Lorenz et al., Phys. Rev. D 92, 034018 (2015). 